\title{
Phase-Encoded Signal Regeneration Exploiting Phase Sensitive Amplification
}

\author{
D. J. Richardson ${ }^{1}$, R. Slavík ${ }^{1}$, J. Kakande ${ }^{1}$, F. Parmigiani ${ }^{1}$, A. Bogris ${ }^{2,3}$, D. Syvridis ${ }^{2}$ and P. Petropoulos $^{1}$ \\ ${ }^{I}$ Optoelectronics Research Centre, University of Southampton, Southampton, SO17 1 BJ, United Kingdom \\ ${ }^{2}$ National and Kapodistrian University of Athens, Panepistimiopolis, Illisia, Athens, GR-15784, Greece \\ ${ }^{3}$ Technological Educational Institute of Athens, Department of Informatics, Aghiou Spiridonos, 12210, Egaleo, Greece \\ Authore-mail address: djr@orc.soton.ac.uk
}

\begin{abstract}
We discuss recent advances in phase-sensitive amplification technology and review its application to the regeneration of multi-level phase-encoded signals.

OCIS codes: (060.4370) Nonlinear optics, fibers; (190.4410) Nonlinear optics, parametric processes; (230.2285) Fiber devices and optical
\end{abstract}

\section{Introduction}

The progression to complex modulation format coding based systems (e.g. MPSK and QAM) presents new opportunities and challenges for all-optical signal amplification, regeneration and processing. The possibilities presented by Phase Sensitive Amplification (PSA) in this regard appear particularly intriguing, offering means of achieving low noise signal amplification [1,2] and optical regeneration [3,4] amongst others. As a consequence considerable research interest has been generated in such topics in recent years - best exemplified perhaps by projects such as the EU Framework 7 project PHASORS, which has reported significant progress in both the underpinning technology required for practical PSA based devices as well as in the applications space itself over the past 3 years [5]. Herein we summarize work directed towards the regeneration of advanced modulation format signals exploiting PSA in highly nonlinear optical fibers (HNLFs). Using suitable combinations of four wave mixing (FWM) processes and injection locking of semiconductor laser diodes we demonstrate "black box" phase and amplitude regeneration of both BPSK and QPSK signals by quantizing the phase at the output of the system to the required number of levels and suitably exploiting amplifier gain saturation.

\section{Principle of Phase Sensitive Amplification and Phase Synchronization}

In a phase-insensitive amplifier, such as an erbium-doped fiber amplifier, the in-phase and quadrature signal components experience identical gain, and as a result the field amplitude is amplified with its phase unaffected (see Fig. 1a).

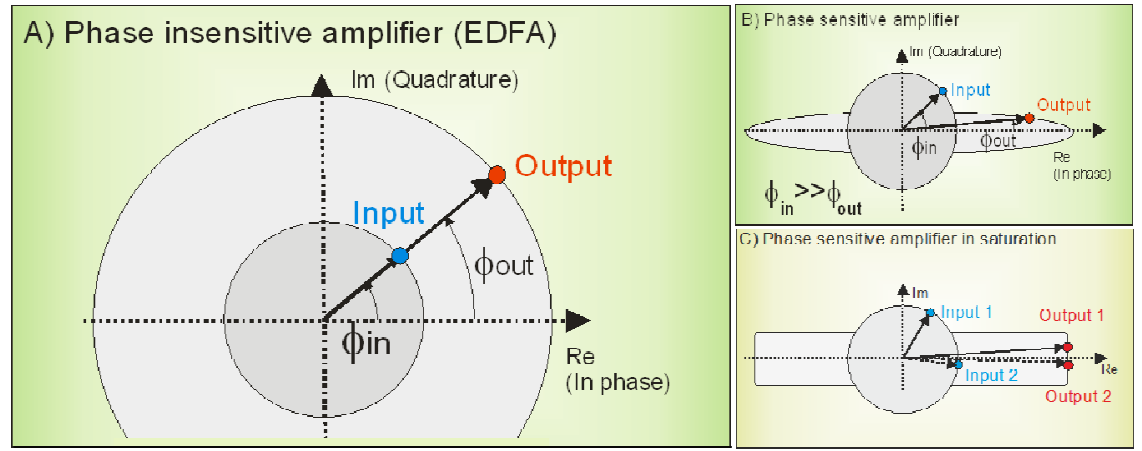

Fig. 1: A phase insensitive amplifier maintains the signal phase (a), while a PSA acts to squeeze its phase (b). To reduce phaseto-amplitude noise conversion it is necessary to clamp the maximum gain e.g. by exploiting gain saturation (c).

However, in a PSA, the two quadrature components are amplified differently; for example, in a PSA based on degenerate FWM as described below, the in-phase component of the electric field experiences gain $g$, while the quadrature component experiences de-amplification by $1 / g$ (see Fig.1b). Subsequently, the output phase of the amplified signal is more closely aligned towards the amplifier's in-phase axis [4]. This effect, known as 'phase squeezing', is inherently suitable for the regeneration of binary phase-encoded signals, as the 'phase squeezed' data bits are forced to adopt a phase of either 0 or $\pi$, in accordance with their phase prior to transmission. Although PSA restores the phase levels of 0 or $\pi$ it is also to be appreciated that it can introduce unwanted amplitude variations. 
This can be avoided by operating the PSA in saturation (see Fig.1c) which can also act to reduce any amplitude noise present on the incident signal, thereby enabling simultaneous phase and amplitude regeneration.

The main challenge in realising practical FWM-based PSA regeneration is to stabilize and maintain a phase relationship between the PSA pump(s) and the signal (and any idlers present) at the PSA input. This is complicated in practice by the fact that phase-encoded signals have a suppressed carrier. Moreover, even if the carrier can be extracted it would generally incorporate any noise generated during data transmission. To overcome these issues schemes have recently been developed based on laser injection locking [6,4] which simultaneously allow carrier recovery and carrier noise suppression. The resulting cleaned carrier can then be used to phase-lock the locally generated pumps with the incoming data prior to signal regeneration in a degenerate two-pump PSA.

\section{Regeneration of BPSK signals}

A schematic of our most advanced embodiment to date of a "black-box" BPSK regenerator based on two pump degenerate FWM is outlined in Fig. 2 [7]. The signal, with noise added either artificially or due to propagation through a real fiber network, is launched directly into the black-box regenerator. First, the signal is combined with a CW local pump ('Pump 1') and sent to a PM-HNLF (HNLF 1) to generate an idler wave that is inherently phaselocked to the data and Pump 1. Then, the three signals (Data, Pump 1, Idler) are demultiplexed in a 4-channel 200$\mathrm{GHz}$ demultiplexer placed behind a circulator. A mirror provides retro-reflection in the data path as well as in the path for Pump 1 which also includes a PZT stretcher and a variable attenuator. A semiconductor laser is injection locked to the idler and used to generate Pump 2 in the idler path. Note that all the optical waves involved in the regeneration process share a common path through the regenerator other than on the output side of the demultiplexer which ensures that they experience similar acoustic/thermal pickup, greatly reducing the environmental sensitivity of the device. The data stream with the two properly locked pumps is then launched into the PSA. For optimum performance the PSA is operated in saturation resulting in a strong variation of pump power at the PSA output as a function of the relative phase between the pump and signal beams. We use this feature to control the PZT fibre stretcher to ensure accurate phase locking over long timescales in the presence of thermal drift.

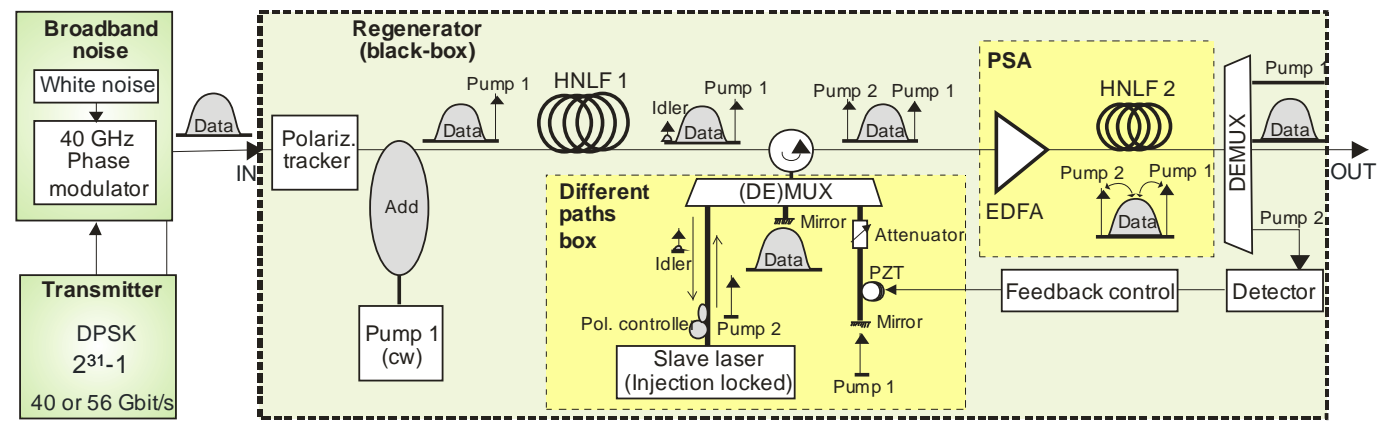

Fig. 2: BPSK regenerator: Experimental set-up.

The performance of the regenerator was first studied using an EXFO constellation analyzer (PSO-200) in the presence of realistic levels of broadband linear and nonlinear phase noise. Results obtained at 40 Gbit/s (see Fig.3) shows that the phase noise can be significantly squeezed with negligible induced amplitude noise [7].

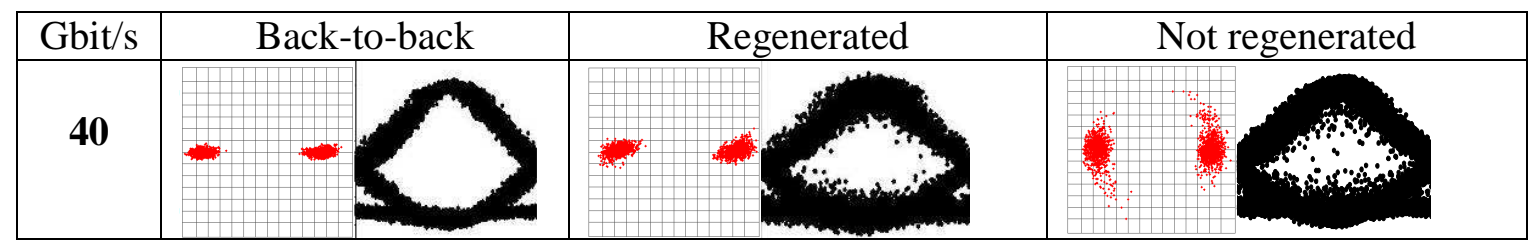

Fig. 3: Constellation diagrams for back-to-back and for added phase noise along with the corresponding demodulated eyes.

Subsequently, we field tested the regenerator in 38 Channel, $40 \mathrm{Gbit} / \mathrm{s}, 100 \mathrm{GHz}$-spacing DWDM transmission experiments over distances of up to $800 \mathrm{~km}$ of installed fibre with the regenerator placed directly in front of the receiver, or in-line at the midpoint of the transmission system. Significant improvements in system performance in accordance with theoretical expectations were obtained in both instances and the robustness/practicality of the regenerator successfully demonstrated [8]. 


\section{Regeneration of MPSK signals}

In order to realize multi-level phase regeneration a more complex stair-case phase transfer rather than a binary phase function is required. We have recently shown that this can be achieved for M-level optical PSK signals by interfering the signal with a conjugated $(\mathrm{M}-1)^{\text {th }}$ phase harmonic in a PSA [9]. As an explicit example QPSK regeneration can be achieved using the setup shown in Fig. 4(top). The QPSK signal is first mixed with a pump to generate a FWM comb of non-degenerate products, including the required $3 \phi$ component. The signal and $3 \phi$ component are then combined inside a dual pump non-degenerate PSA where coherent addition is obtained. Of the two required pumps, the first is derived from the free running laser originally used in the FWM comb generation stage, and the second by injection locking a semiconductor laser to the $4 \phi$ (modulation stripped) FWM component, thereby satisfying the phase locking requirement. Examples, of QPSK regeneration at various baud rates for different levels of phase and amplitude noise are shown in Fig.4 (bottom) validating the approach.

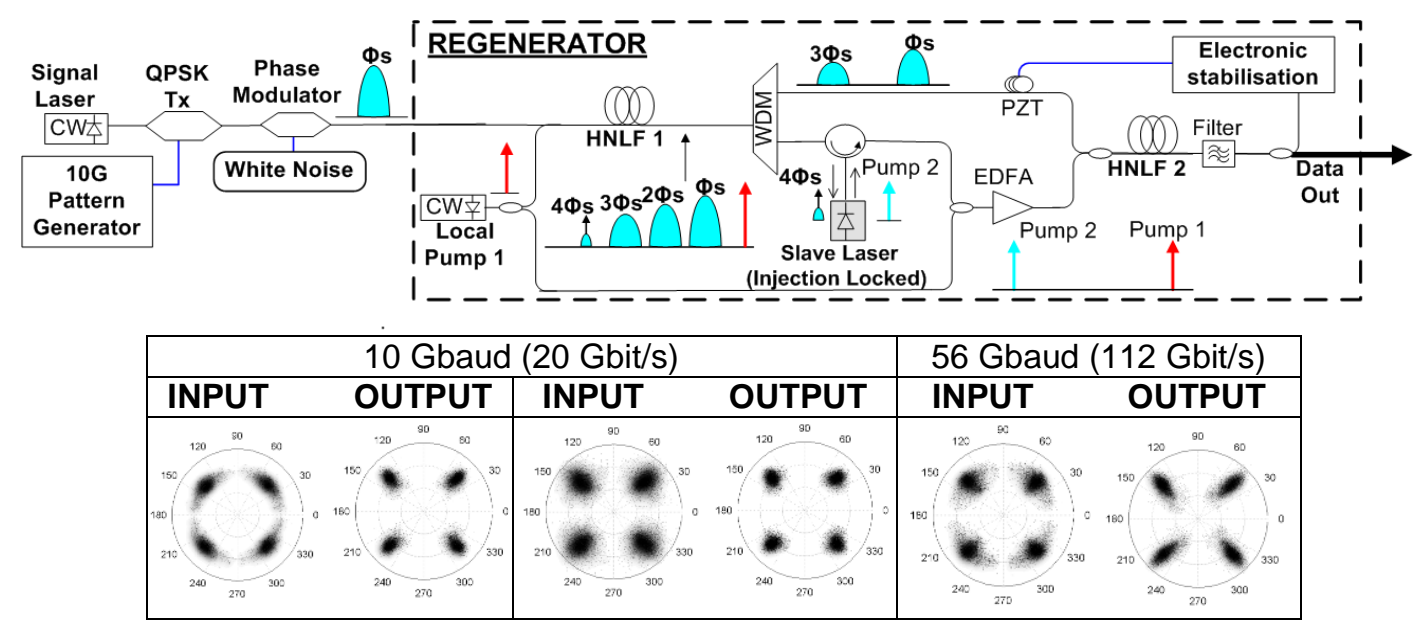

Fig. 4: (top) Schematic of QPSK regenerator, and (bottom) input and output constellation data at 10 and 56 Gbaud for various input noise distributions (obtained using a slightly modified embodiment of Fig.4 (top)).

\section{Conclusions}

In conclusion, major developments in PSA technology and concepts have resulted in significant advances in the optical regeneration and processing of advanced modulation format signals allowing detailed studies of subsystem and overall system performance in the presence of both linear and nonlinear phase noise. Further advances in terms of extension to multi-wavelength operation and the regeneration of more complex modulation formats, e.g. QAM, are eagerly anticipated.

\section{Acknowledgements}

We would like to thank OFS Fitel Denmark for providing the silica HNLFs and Eblana Photonics for the supply of discrete mode lasers used in these experiments. This research has received funding from the European Communities Seventh Framework Programme FP/2007-2013 under grant agreement 224547 (PHASORS) and from the UK Engineering and Physical Sciences Research Council under grant agreement EP/I01196X/1 (Hyperhighway).

\section{References}

[1] C.M. Caves, "Quantum limits on noise in linear amplifiers", Phys. Rev. D 26, 1817-1839 (1982).

[2] Z. Tong et al.,"Towards ultrasensitive optical links enabled by low-noise phase-sensitive amplifiers", doi:10.1038/nphoton.2011.79, (2011).

[3] K. Croussore \& G. Li, "Phase regeneration of NRZ-DPSK signals based on symmetric-pump phase-sensitive amplification", IEEE Photon. Technol. Lett. 19, 864-866 (2007).

[4] R. Slavik et al., "All-optical phase and amplitude regenerator for next-generation telecommunications systems", Nature Photonics, Vol.4 pp.690-695 (2010)

[5] See http://www.eu-phasors.eu/index.html

[6] Weerasuriya, R. et al., "Generation of frequency symmetric signals from a BPSK input for phase sensitive amplification", in Proc. OFC 2010, San Diego, USA, paper OWT6 (2010).

[7] R. Slavik et al., "Robust design of all-optical PSK regenerator based on phase sensitive amplification", Proc. OFC 2011, paper OMT2, (2011).

[8] R. Slavík et al., "Field-trial of an all-optical PSK regenerator in a 40Gbit/s 38 channel DWDM transmission experiment", Proc. OFC 2011, PDPA7 (2011).

[9] J. Kakande et al., "First demonstration of all-optical QPSK signal regeneration in a novel multi-format phase sensitive amplifier", Proc. ECOC 2010, PD3.3, (2010). 